\title{
Stage III Lung Cancer AJCC v8
}

National Cancer Institute

\section{Source}

National Cancer Institute. Stage III Lung Cancer A/CC v8. NCI Thesaurus. Code C136478.

Stage III includes: IIIA: (T1a, N2, M0); (T1b, N2, M0); (T1C, N2, M0); (T2a, N2, M0); (T2b, N2, M0); (T3, N1, M0); (T4, N0, M0); (T4, N1, M0); IIIB: (T1a, N3, M0); (T1b, N3, M0); (T1c, N3, M0); (T2a, N3, M0); (T 2b, N3, M0); (T3, N2, M0); (T4, N2, M0); IIIC: (T3, N3, M0); (T4, N3, M0). T1a: Tumor measuring $1 \mathrm{~cm}$ or less in greatest dimension. $A$ superficial, spreading tumor of any size whose invasive component is limited to the bronchial wall and may extend proximal to the main bronchus also is classified as T1a, but these tumors are uncommon. T1b: Tumor measuring more than $1 \mathrm{~cm}$ but $2 \mathrm{~cm}$ or less in greatest dimension. T1c: Tumor measuring more than $2 \mathrm{~cm}$ but $3 \mathrm{~cm}$ or less in greatest dimension. T2a: Tumor measuring more than $3 \mathrm{~cm}$ but $4 \mathrm{~cm}$ or less in greatest dimension. T2 b: Tumor measuring more than $4 \mathrm{~cm}$ but $5 \mathrm{~cm}$ or less in greatest dimension. T3: Tumor measuring more than $5 \mathrm{~cm}$ but $7 \mathrm{~cm}$ or less in greatest dimension or directly invading any of the following: parietal pleura (PL3), chest wall (including superior sulcus tumors), phrenic nerve, parietal pericardium; or separate tumor nodule(s) in the same lobe as the primary. T4: Tumor measuring more than $7 \mathrm{~cm}$ or tumor of any size invading one or more of the following: diaphragm, mediastinum, heart, great vessels, trachea, recurrent laryng eal nerve, esophagus, vertebral body, or carina; separate tumor nodule(s) in an ipsilateral lobe different from that of the primary. N0: No regional lymph node metastases. N1: Metastasis in ipsilateral peribronchial and/or ipsilateral hilar lymph nodes and intrapulmonary nodes, including involvement by direct extension. N2: Metastasis in ipsilateral mediastinal and/or subcarinal lymph node(s). N3: Metastasis in contralateral mediastinal, contralateral hilar, ipsilateral or contralateral scalene, or supraclavicular lymph node(s). M0: No distant metastasis. (AJCC 8th ed.) 